\title{
Le désordre du monde
}

\author{
Babacar Ndiaye*
}

\section{Résumé}

Le début du XXIe siècle se caractérise par l'accumulation et la consécution vertigineuse d'événements graves : crises, actes terroristes aveugles et guerres. Cet état de fait est au fondement d'une analyse critique, sans pessimisme ni complaisance, de la situation qui prévaut dans le monde. Celle-ci s'apparente profondément à un désordre. Par-delà ce constat et en guise de simple contribution, quelques pistes indicatives sont explorées pour la résorption de ce dérèglement du monde.

\begin{abstract}
The beginning of the twenty-first century is characterized by the exponential accumulation succession of serious events: crises, indiscriminate terrorist acts and wars. Void of any pessimism or complacency, this article constitutes the foundation of a critical analysis of the situation prevailing in the world. This deeply resembles disorder. Beyond this acknowledgement, and as a simple contribution, some indicative tracks are explored for the elimination of this world disorder.
\end{abstract}

La chute du mur de Berlin (1989) avait suscité un immense espoir de libération, d'ouverture et d'évolution convergente de tous les peuples. La fin du paradigme de la guerre froide laissait donc présupposer des lendemains meilleurs et enchanteurs avec un nouvel ordre stable.

Or, à ce jour, le monde s'ancre davantage dans l'instabilité, l'absence de traçabilité de sa trajectoire quasi-erratique, ainsi que dans l'inexistence

* Nous sommes malheureusement au regret d'annoncer que Babacar Ndiaye nous a quitté. Il est mort dans l'attentat d'Alger contre les Nations unies, le 11 décembre 2007. Nous publions son texte en l'état, sauf corrections mineures.

We are unfortunately sorry to announce that Babacar Ndiaye has passed away. He was one of the victims who died in the attack on the United Nations office in Algiers, on 11 December 2007. We publish his text as it was written, except for minor corrections'. 
chronique d'un vrai modèle référentiel accepté et réalisable à court ou moyen terme. Déjà, Maurice Druon, dans un aperçu de l'état du monde, souhaitait « inviter tout d'abord à considérer notre monde tel qu'il est [...] monde à la fois élargi et rétréci, divisé et lié [...]. Rétréci, par les communications qui mettent tous les peuples en contact immédiat, visuel, auditif ou physique, les uns avec les autres. Monde divisé par les désordres, les conflits, les affrontements ethniques, les rivalités historiques, économiques, religieuses, idéologiques. Mais monde lié également par ses besoins, ses craintes, ses aspirations ».

En fait, il semble s'instaurer et s'instituer, sous les apparences d'une mauvaise passe, un système protéiforme synonyme d'un capharnaüm, logiquement et moralement inconcevable au XXIe siècle.

Le désordre caractéristique de notre monde est une surprise permanente dont les modalités de gestion ne sont pas aujourd'hui des plus appropriées et qu'il convient de résoudre au plus vite.

\section{La surprise au quotidien}

Au regard des événements récurrents qui secouent le monde, la surprise au quotidien est de mise. Le choc et la panique sont d'actualité et le mettent à rude épreuve du fait de nouvelles dérives, des modes d'actions extrêmes employés, voire même des avatars de la mondialisation.

\section{Les nouvelles dérives surprenantes}

Le troisième millénaire débute par le choc et l'accélération d'une violence anormale. Il maintient en émoi les populations, directement ou indirectement. À ce rythme d'accumulation de dérives, le monde glisse inexorablement vers l'abîme, loin de ses références habituelles.

D'emblée, tout porte à croire que le monde évolue sans repères justes, ni fiables. De nouvelles dérives semblent s'installer durablement dans la conduite des affaires, sans qu'il soit possible de les freiner ou de les arrêter immédiatement. En effet, la situation, en perdurant, devient endémique. Mais c'est plutôt son degré d'acuité ainsi que ses manifestations déconcertantes qui posent problème. Ainsi, aux mensonges d'État succèdent des exécutions extrajudiciaires orchestrées à des niveaux très élevés ainsi que des manipulations inadmissibles de l'information à des fins électoralistes.

De même, face aux agissements peu orthodoxes des gouvernants, les citoyens ont l'immense impression de subir une sorte de manipulation et de hold-up de leur délégation de pouvoir. Ils perdent toute emprise sur les mandataires. La démocratie directe n'étant pas possible en permanence, ils subissent régulièrement les décisions qui ne rencontrent pas leur aval. Par 
conséquent, entre les rendez-vous périodiques et espacés des consultations électorales, il leur reste la rue pour déverser leur rancœur, leur opposition, voire leur défiance vis-à-vis des décideurs pourtant investis en leur nom pour un contrat à durée déterminée renouvelable à échéance. Le rythme auquel les manifestations se succèdent à travers le globe, dans les rues des grandes villes, pour des causes souvent légitimes, montre combien les dérives surprenantes envahissent le monde et poussent les individualités et les organisations à réagir. C'est de cette manière que la surprise traduit une réalité constante qui rythme la vie, surtout par rapport à des questions d'envergure ou à des enjeux décisifs, comme la guerre, touchant la paix et la sécurité du monde.

Donc, les nouvelles dérives prennent les citoyens en otage et contribuent à déstabiliser un monde en mal de repères. Certaines options s'orientent délibérément vers le chaos pour atteindre des buts hypothétiques par le biais d'un cap incertain et problématique. Tout cela est à l'image de certains modes d'action inconcevables à l'œuvre.

\section{Des modes d'action inconcevables}

À l'évidence, le monde apparaît comme un grand théâtre d'affrontements. Il ne s'agit en aucun cas du paradigme du choc prédit suivant les hypothèses huntingtoniennes qui réduisent le monde en un gigantesque champ de bataille selon les lignes de front culturelles. Au contraire, si choc il y a, c'est un choc à rebours et par surprise, suivant en cela les choix des États et les options des groupuscules non-représentatifs. Alors, ces adversaires réels ou potentiels s'emploient à des modes d'action différents, mais dont le dénominateur commun est, parfois dans des cas précis, leur irrecevabilité.

Certes, le terrorisme érigé en stratégie de déstabilisation du monde reste inconcevable et moralement injustifiable. En tant que mode de destruction brutale, souvent suicidaire, il se trouve en dehors de la sphère de l'affrontement direct et normé qui convient dans les cas d'opposition ou de différends entre parties. Les résultats catastrophiques que le terrorisme produit, en termes de bilan macabre et d'impact psychologique, en font un mode d'action illégitime. Or, dans le monde d'aujourd'hui, le terrorisme s'ancre davantage dans les mœurs déshumanisées de beaucoup de franges marginales extrémistes qui se développent un peu partout. En témoignent la fréquence élevée des actions terroristes ainsi que les bilans toujours plus lourds enregistrés depuis les attentats du 11-Septembre jusqu'à celui de Madrid, en passant par ceux de Bali, Casablanca, Djerba, Alger, Istanbul, etc. Face à ce fléau en pleine expansion, la réaction des États s'organise de diverses façons. 
De fait, il faut barrer la route, voire inverser la tendance du développement $\mathrm{du}$ terrorisme et des tentatives de son érection comme modèle efficace d'institution de la peur panique dans les sociétés démocratiques. En revanche, parmi tous les modes d'action en œuvre, l'un des plus contestables demeure l'idée même de guerre préventive pre-emptive. Les mesures guerrières anticipatives et punitives faussent l'idée même de légitimité et de justice qui doivent, en principe, accompagner toute action de coercition de force. Pourtant, dans ce cas ciblé, le droit de la force brute semble l'emporter sur la force du droit équitable, dans la mesure où l'évacuation des éléments matériels et l'absence de base légale privent toute guerre préventive de fondements fiables. En conséquence, tout en essayant de régler certains problèmes bien localisés, ces types d'actions programmatiques renforcent le développement de sentiments négatifs puissants déjà bien ancrés dans certaines populations et régions du monde, en dépit de la direction contraire de la stratégie d'influence américaine. Dans ce contexte, l'action étatique s'assimile de facto à une modalité impropre, parce que porteuse de germes qui laissent présager une inscription du problème supposé réglé dans la durée. La guerre en Irak, par exemple, a un impact certain sur le développement exponentiel du sentiment anti-américain déjà porté très haut par la question palestinienne. En tant qu'expression la plus achevée du hard power, elle ne rencontre pas l'assentiment de toute la communauté internationale qui ne peut que se résigner ou manifester son désaccord, souvent à travers « un état permanent d'hypocrisie diplomatique $»$.

En définitive, ces deux figures aux antipodes l'une de l'autre et massivement mises en œuvre dans le contexte présent, dessinent des modes d'action qui ne peuvent recevoir qu'une caution marginale. Au regard de la situation actuelle et des faits en cours à travers la géographie des conflits, telles sont les modalités pratiques les plus médiatisées qui gouvernent la déstabilisation profonde et le désordre du monde. D'une manière moins brutale, la mondialisation contribue aussi au désordre du monde.

\section{Les avatars de la mondialisation}

A priori, la mondialisation est porteuse du principe d'espérance. Elle est la dynamique qui incarne la nouvelle donne au plan économique et relationnel. Concrètement, la mondialisation se traduit par des échanges massifs, la vitesse des procédures et de l'information à travers la planète, etc. Néanmoins, la mondialisation est aussi le lieu de l'ambivalence. Elle a ses forces, mais également ses grandes faiblesses, dont l'exclusion contre lesquelles se bat le mouvement altermondialiste. 
En premier lieu, la mondialisation crée une situation d'exclusion sans précédent. En effet, l'emprise procédurière des grandes firmes internationales sur les échanges dope considérablement le libéralisme outrancier. Il y a une sorte d'hégémonie du libéralisme, avec ses instances supranationales de direction que sont le Fonds monétaire international et la Banque mondiale. De ce fait, les États ont perdu une certaine maîtrise de leur propre économie désormais rivée à la vitesse supersonique de la mondialisation. Par conséquent, suivant les lois du marché mondial et ses exigences statistiques, il y a une profonde modification de la donne intérieure en matière économique, et par extension dans le domaine social. Le contre-coup de cette dynamique nouvelle se fait sentir au niveau des collectivités locales et humaines périphériques et donc forcément au plan individuel. C'est pourquoi, le mouvement altermondialiste a vu le jour et se développe de façon vertigineuse, dans le but de faire face aux avatars de la mondialisation, en tant que forme de contrepouvoir d'envergure planétaire.

En second lieu, l'exclusion est cette création de zones géographiques et économiques quasi-étanches. Bien que le vœu pieux eût été de réaliser un bond qualitatif et quantitatif de tous les pays et de la société mondiale vers la modernité ou la postmodernité, ce pari semble d'emblée voué à l'échec. En réalité, cette prouesse n'est pratiquement pas réalisable. La preuve se révèle simplement des échecs répétés constatés lors des deux dernières assises de l'Organisation mondiale du commerce (OMC). Le diktat subtilement imposé par les pays du Nord s'est vu opposé un refus catégorique des pays du Sud. La raison tient au fait que les conditions proposées allaient dans le sens de l'asphyxie économique des pays en développement. Or, la volonté de niveler vers le haut aurait exigé que des efforts d'encouragement, des pratiques favorables et préférentielles, ainsi qu'une volonté de combler le retard de certains pays par une valorisation de leurs ressources soient à la base des propositions d'accord. Tout laisse à penser que la volonté était inexistante. Ainsi, ce côté de la mondialisation approfondit la rupture entre le Nord et le Sud, maintient le fossé économique et pérennise le cloisonnement géographique préjudiciable à toute idée de communauté globale intégrée. Sous les apparats de l'isotropie, se trouve en réalité un véritable rideau de fer sous tension sur lequel viennent régulièrement s'échouer, malheureusement, des flots d'immigrés clandestins fuyant la misère.

Ainsi, par certains côtés, la mondialisation contribue au désordre du monde. Elle participe au maintien d'un écart, voire d'une partition de fait entre les États, au lieu de traduire en acte l'interconnexion des différentes entités d'un monde uniforme et isochrone. 
Les nouvelles dérives mettent le monde dans une situation d'urgence endémique. Les réponses apportées emploient des modes d'action inappropriés. De son côté, la mondialisation crée, dans son sillage, l'exclusion. Toutes ces raisons maintiennent un état permanent de surprise. Le rôle du leadership mondial devrait, en principe, participer à la régulation du monde par une gestion plus équitable et moins sujette à controverses. Force est de constater que cela n'a pas été le cas jusqu'ici.

\section{Leadership et gestion chaotiques du monde}

De prime abord, le poids économique, politique et militaire de certains États leur confère d'office un rôle primordial dans la guidance des relations internationales. Ce leadership indéniable se décline aussi en terme de responsabilité en matière de sécurité, de stabilité et de tranquillité dans le monde. Or, le XXe siècle et son prolongement en ce début de XXIe siècle se révèlent meurtriers et chaotiques au regard des guerres démultipliées, des risques encourus et des dangers auxquels les populations de tous bords sont en permanence exposés (maladie, famine, drogue, pollution, terrorisme, etc.). Si la responsabilité reste globale et partagée, elle se définit au prorata des capacités plus ou moins considérables des entités étatiques, et par conséquent au regard de leur pouvoir d'orientation, d'influence, d'infléchissement et d'intervention sur le cours des événements, au profit exclusif de la communauté humaine dans son ensemble. Dans les faits, cette vision idéale, naïve et candide, se heurte violemment au mur de l'unilatéralisme, des modèles inadaptés et des intérêts nationaux hypertrophiés.

\section{Un unilatéralisme dangereux}

Le danger de l'unilatéralisme se situe au moins à deux niveaux distincts et complémentaires : d'un côté, il crée le désordre par ses procédés très peu consensuels, de l'autre il exacerbe les passions proportionnellement au désordre induit.

D'abord, la position privilégiée de leader ne se traduit pas systématiquement en attitude démocratique. Elle a plutôt la maladroite tendance à se métamorphoser en une ferme volonté de puissance dont la manifestation la plus actualisée est l'unilatéralisme. Celui-ci se traduit en une imposition de volonté, de choix et d'options sans tenir compte des autres ou de l'intérêt global. Il consiste à se refuser à tout débat contradictoire et surtout à toute prise en compte d'opinions divergentes ou contraires. Au nom de la force que confère le leadership, il y a une sorte de confusion volontaire entre l'intérêt particulier et la volonté générale qui débouche inexorablement sur des orientations critiquables, des choix exclusifs et controversés et des décisions 
minoritaires. Tout cela contribue donc à l'éclosion et à l'entretien du désordre à l'échelle planétaire. L'illustration la plus récente de cet unilatéralisme en acte est l'échec des discussions au Conseil de sécurité de l'ONU au sujet du déclenchement de la guerre contre le régime de Saddam Hussein : les ÉtatsUnis se sont affranchis du cadre onusien et ont fait fi du désaccord ouvert de la France, de la Chine et de la Russie. Contrairement à la première guerre du Golfe de 1991, qui avait reçu l'assentiment et le soutien de tous, ainsi que la double légitimité - celle découlant de l'agression et celle de la résolution de l'ONU_, la guerre de 2003 a été fondée sur une vision unilatérale. Cette forme de leadership participe au désordre international et crée par ailleurs des précédents graves et dangereux.

Ensuite, les modalités unilatérales de décision sur des questions d'enjeu global contribuent également à l'entretien du désordre mondial. Par la démultiplication des déçus et l'apparition de nouveaux mécontents, l'unilatéralisme fertilise continuellement le terreau du radicalisme "terroriste" qui menace la paix. En reflétant l'injustice, la force brute et une hégémonie flagrante, l'unilatéralisme participe à l'éclatement du monde et à l'antagonisme perpétuel de groupes qui auraient pu coexister pacifiquement. Malheureusement, tout semble indiquer que la cécité et la préservation d'intérêts ou d'alliances guident les choix qui ne font qu'exacerber les sentiments d'abandon, de mépris et d'injustice. C'est dans ce cadre que s'interprète le soutien isolé et très marginal au plan de retrait des territoires palestiniens de Gaza par les hautes instances politiques américaines. Avec un tel scénario, le leadership attendu d'une grande puissance est non seulement biaisé, mais il est surtout le catalyseur d'une rupture de confiance et l'ouverture vers des voies extrêmes de confrontation avec l'adversaire.

L'unilatéralisme est une tentation qui met en péril le monde, à l'instar des modèles pas toujours adéquats proposés pour assainir un monde en proie au désordre.

\section{Des modèles inadaptés et rejetés}

Parmi les échecs qu'enregistre le leadership planétaire, il y a effectivement le refus de modèles inadaptés censés enrayer le désordre mondial. Toutefois, ces exemples savamment forgés par des experts en stratégie et portés sur les fonds baptismaux par les voix politiques autorisées se révèlent non seulement arbitraires, mais aussi irrecevables par leurs destinataires. Il en est ainsi de certains projets de remodelage géopolitique et de catégories référentielles appliquées à l'analyse de la situation du monde.

La sortie de crise passe nécessairement par un remodelage géopolitique du monde. Le modèle par excellence combine à la fois, par un savant dos- 
age, la démocratie et le libéralisme. Aujourd'hui, les locomotives du leadership mondial érigent la bonne gouvernance, la démocratie et l'économie de marché en critères absolus et indices spécifiques auxquels les États doivent s'arrimer à l'intérieur des grands ensembles sous-régionaux et continentaux. C'est un rêve réalisable. En revanche, il peut se changer en cauchemar, car la remise en cause accélérée de l'immobilisme de certaines sociétés pourrait bien se révéler une entreprise périlleuse. Une démarche processionnelle qui prendrait le temps utile à l'ancrage solide des réformes dans les habitudes aurait certainement plus de chance de succès. Dans la mesure où ce choix s'inscrit dans la durée, il y a moins de risques de tomber dans des travers insoupçonnés et non-souhaitables. Le projet américain du Grand MoyenOrient (GMO) relatif aux pays arabo-musulmans, en dépit du soutien de partenaires inconditionnels, ne fait pas l'unanimité. Au contraire, cette initiative étrangère est en bute à un refus catégorique de l'Égypte, de la Jordanie et de l'Arabie saoudite notamment. Mort-né, ce projet a de fortes chances de se ranger parmi les modèles inadaptés.

En plus de l'inadaptation de certains modèles, des catégories référentielles posent de nombreux problèmes. En effet, du diagnostic de la crise sécuritaire il ressort des qualifications de l'état des choses et de ses causes. Le grand risque de cette conceptualisation est le glissement de sens, l'interprétation abusive et surtout la confusion avec d'autres champs de rationalité. C'est pourquoi la prudence devrait être de mise en la matière, afin d'éviter les dérapages lexicologiques et sémantiques qui seraient inexorablement exploités à contre-courant. En réalité, dans un monde de communication et d'information, en plus de la diversité culturelle, toute référence catégorielle mérite une analyse sérieuse. Par exemple, les catégories de Bien et de Mal sont très relatives. Elles dépendent de l'optique propre et du cadre d'émission. De surcroît, elles ont généralement une connotation plus religieuse et morale que politique. Et c'est là où réside principalement le grand risque de confusion. Par rapport aux extrémismes qui se drapent d'atours religieux, brandir de telles catégories c'est plus ou moins les rejoindre dans leur sphère d'intelligibilité ou, au moins, les renforcer dans leur intime conviction. C'est aussi la raison pour laquelle ces références ne sont pas strictement appropriées. Plus qu'à l'époque de Nietzsche et de Heidegger, l'humanité a besoin de cheminer par-delà le bien et le mal, sinon le monde risque de s'engouffrer dans le dédale des chemins qui ne mènent nulle part.

Un modèle préfabriqué, qui de plus l'est du dehors, ne peut s'imposer à quelque région ou peuple. La recherche du compromis, de l'adhésion et de la participation sont des atouts qui peuvent permettre d'éviter des écueils, un éternel recommencement et surtout de faux départs sur la voie de 
l'ordonnancement du monde. Tel serait un des facteurs déterminants d'un monde comme système harmonieux, par-delà le primat exclusif des intérêts nationaux.

\section{Le primat des intérêts nationaux}

La hiérarchie des préférences place logiquement les intérêts nationaux avant toute autre considération. Ce choix est un des soubassements de la géopolitique et de la géostratégie. Cependant, dans bien des domaines spécifiques que le monde a en partage, les intérêts nationaux particuliers ont moins d'importance que l'effort collectif de résolution des problèmes qui se posent; car dans de tels cas de figure, les voix des experts et des organisations internationales devraient non seulement convaincre, mais aussi susciter l'adhésion. Cela n'est pas toujours l'option privilégiée.

D'une part, la stratégie d'influence des empires modernes est intimement liée à leurs préoccupations et à leurs intérêts nationaux. Au risque de transgresser les règles élémentaires des relations internationales, il est souvent fait obstruction à la norme pour atteindre des objectifs politiques, économiques et stratégiques. Le primat de ce type d'intérêt induit le désordre. Mieux, il consacre le règne et le triomphe du chaos. En effet, il y a un minimum de respect des règles communes pour que l'ordre puisse exister et régir les relations entre les États. Si certains transgressent les dispositions communes, qu'elles soient écrites, coutumières ou basées sur la jurisprudence, le désordre se substitue à l'ordre, au modèle et à l'exemplarité. Pourtant, il est du ressort des puissances de montrer la voie, de donner le bon exemple et de servir de référence. Or, il n'en est pas ainsi chaque fois que le besoin se fait sentir. À ce titre, l'entrée en vigueur de la Cour pénale internationale (CPI) a montré comment la primauté des intérêts nationaux a failli bouleverser profondément l'ordre précaire du monde, par le non-renouvellement du mandat des missions de maintien de la paix. Et pourtant la nécessité d'une justice internationale compétente et partagée par tous ne peut que s'inscrire dans l'optique de l'assainissement du monde. De même, sur le plan environnemental, la nonratification du protocole de Kyoto contribue à pérenniser une certaine forme de désordre que d'autres États pourraient librement transposer, en réplique, dans la non-signature d'autres traités et conventions.

D'autre part, cette stratégie délibérée de blocage se dédouble d'une imposition de la puissance. La mainmise qui tente de s'exercer sur les ressources stratégiques s'inscrit dans cette veine. Aujourd'hui, la géostratégie semble opérer un glissement rapide par une transmutation et une occupation du domaine géo-énergétique. La logique du contrôle des principales sources de production du pétrole conditionne la stabilité du monde. De la plus ou moins 
grande facilité à contrôler l'or noir dépend le degré plus ou moins important d'ordre dans les régions concernées. Ainsi, le Moyen-Orient, en tant que première région de production pétrolière, est entré en effervescence depuis la première guerre du Golfe. Cette instabilité a une origine bi-univoque : si elle n'est pas le fait de puissances hégémoniques qui vont à la recherche de la précieuse matière première, c'est à cause des extrémismes s'opposant à la fois à ces marchés et à l'oppression des régimes tyranniques qui en bénéficient. Pour ainsi dire, le primat des intérêts nationaux est un puissant levier qui interfère dans le désordre du monde.

Globalement, une négligence des aspects collectifs planétaires par rapport à des intérêts nationaux toujours surdéterminés conduit à l'entropie. Cela débouche sur le désordre, au regard des questions qui nécessitent un consensus, un accord et une démarche collective sans (auto) exclusion.

Tout compte fait, la problématique du leadership et de la gestion du monde offre une image chaotique. Elle ne semble obéir à aucune règle. Alors, se pose une difficulté majeure à lire objectivement la trajectoire des États pris singulièrement au sujet de la suite réservée aux enjeux collectifs et supranationaux. C'est la raison fondamentale pour laquelle l'urgence d'une thérapie s'impose pour sauver le monde, s'il reste vrai que celui-ci est toujours un système, au sens physique du terme.

\section{Une thérapie urgente et nécessaire}

Le désordre du monde implique, dans une visée moins critique et plus constructive, une thérapie placée sous le double signe de l'urgence et de la nécessité. En effet, le génie humain peut infléchir la situation et la ramener à des proportions moins dramatiques. Il s'agit donc de freiner la tendance erratique observable et de déterminer des bases claires, stables et acceptables qui puissent réintroduire l'harmonie et surtout la lisibilité dans les relations internationales. Comme pendant les phases délicates et les seuils critiques déjà atteints, le sursaut est possible. Sa probabilité dépend de certains facteurs de pondération, comme la spécificité locale et l'idée de justice, ainsi que de la place accordée aux personnes morales dont l'ONU est l'incarnation la plus adaptée, pour le moment, sous réserve de quelques réformes et d'un bon usage.

\section{L'incontournable prise en compte des spécificités}

De façon pratique, le monde comme système ne saurait véritablement ignorer les particularités propres aux différentes régions, aux entités nationales et surtout aux peuples. La spécificité, au lieu d'être un facteur de scission, est plutôt un élément de pondération qui sied bien à la diversité caractéristique 
des composantes du puzzle planétaire. En tant qu'élément incontournable, elle s'apprécie à deux niveaux.

D'un côté, il y a la dimension pragmatique. En fait, à la place du modèle précuit qu'il s'agirait de plaquer de facto à une situation donnée, il suffit simplement de puiser dans les possibilités locales parmi les plus proches des standards et surtout les plus adaptées au contexte en question. Tout en étant pratique, cette option, plus soft et moins frustrante, offre l'opportunité d'une certaine forme de familiarité et de proximité. Elle bouleverse moins les us et coutumes, ainsi que les sentiments et les convictions. Ce pragmatisme donne même l'impression d'une reconnaissance de valeurs spécifiques ainsi que leur valorisation au-delà des limites géographiques et culturelles de la nation ou des populations concernées. Deux illustrations confirment cette optique. D'abord l'implication des structures traditionnelles afghanes dans l'élaboration de la loi fondamentale, suite à la défaite du régime des talibans. Cette implication a certainement permis d'éviter la germination d'un sentiment d'imposition, et par conséquent d'une attitude de rejet. Ainsi, tout en étant proche d'une démocratie directe, car les délégués à la Loya Jirga sont représentatifs de leur tribu, cette mise en valeur d'une spécificité locale offre presque tous les avantages d'une démocratie réelle et reste conforme au souhait de réformer la société considérée. Ensuite, les Gacaca rwandais donnent l'exemple d'une particularité locale exploitée à bon escient. Quels que soient leurs valeurs et leurs résultats, ces tribunaux traditionnels contribuent à la fois à la manifestation de la vérité et au processus de reconstitution du tissu relationnel entre Tutsi et Hutu. Eu égard à l'ampleur du génocide, au nombre très élevé de personnes impliquées à divers degrés, et surtout à l'impérieuse nécessité de réconcilier la nation avec elle-même, cette contribution ne saurait être négligeable.

De l'autre côté, il y a la dimension incompressible. La culture reste une forme on ne peut plus achevée de la spécificité. Ainsi, loin d'être une source de division, une ligne de front et un facteur polémogène, elle peut être constitutive d'intégration. Du fait des contacts inévitables entre cultures, il y immanquablement échange, dépassement, progrès par synthèse, assimilation et finalement enrichissement réciproque. Si tant est qu'en la matière aucune culture ne puisse se développer en autarcie, la rencontre et le dialogue interculturel apportent une plus-value comme contribution à la paix et à la stabilité du monde. Les particularités locales ont donc un rôle à jouer. Cela se traduit concrètement en un apport à la civilisation de l'universel, dans la lignée de l'humanisme de Pierre Teilhard de Chardin et du cosmopolitisme cher à Léopold Sédar Senghor. Aujourd'hui, la Francophonie constitue le modèle d'espace de convergence culturelle. Elle a, à la fois, une dimension 
culturelle, voire interculturelle et des aspects académiques et politiques sans conteste. « La Francophonie est ainsi le lieu d'une certaine forme d'altérité positive, non pas verticale, mais horizontale, parce qu'espace du brassage et du métissage culturel qui doivent déboucher sur une personnalité nouvelle, une personne morale imposante sur l'échiquier international et un dialogue égalitaire multilatéral de dimension mondiale ». Autrement dit, la culture peut et doit contribuer à la paix et à l'ordre.

Au final, la contribution de toutes les entités s'avère indispensable pour rétablir l'ordre du monde. En puisant dans les particularités et dans la culture de chaque partie, il est possible de remédier au désordre constaté par la création de nouvelles valeurs et de références globalement partagées. C'est de cette manière que se construit l'identité de l'humanité par la balance.

\section{La nécessité d'une justice internationale}

Afin de mieux réguler le monde, il faut obligatoirement inviter ou forcer toutes les entités à respecter les règles communes. La nécessité d'une justice se fait plus sentir au plan international, car elle enraye la réalité selon laquelle le monde est à géométrie variable. Aussi longtemps que sous le même rapport et dans les mêmes conditions deux faits identiques et produisant le même effet ne seront pas traités de la même manière, il y aura peu de chance d'ordre dans le monde. Cet ordre est incarné par la justice internationale, au propre comme au figuré.

Au propre, la justice est dite dans les tribunaux, les cours et les chambres. Lorsque toute tentative de règlement à l'amiable ou par médiation échoue, le droit doit être dit et rigoureusement appliqué. Suivant cette optique, il n'y a que la justice qui puisse permettre d'atteindre cet objectif. En effet, elle seule est en mesure d'extirper le droit de la force. Mais encore faut-il que tous y adhèrent sans réserve pour permettre de partir sur des bases communes. Hélas, tel n'est pas le cas aujourd'hui et on en est encore loin. Pourtant, la justice a pris une place primordiale dans la liquidation des grands contentieux du siècle passé. Pour preuve, le tribunal spécial institué après la Seconde Guerre mondiale ou les tribunaux spéciaux pour l'ex-Yougoslavie (TPIY) et le Rwanda (TPIR) qui continuent de juger ces passifs récents. Mieux, l'ONU a dans ses démembrements la Cour internationale de Justice de La Haye. Certes son domaine de compétence est limité et ses modalités de saisine sont trop formelles mais il reste que cette cour contribue à la justice dans le monde. Face au cadre restreint de ses matières spécifiques, la CPI a été instituée avec peine et grand bruit. La réticence manifestée par les ÉtatsUnis a présidé à la naissance aux forceps de cet outil d'équilibre du monde en juillet 2002. Eu égard à ce handicap de taille, il est même loisible de s'interroger 
sur l'efficacité attendue de cet instrument, si le gendarme du monde refuse de se plier à ses compétences.

Au figuré, la justice devrait sous-tendre l'action de tous, sans exception. Cette justice non-écrite contribue à la suppression des paradoxes flagrants qui se lisent dans le monde. C'est elle qui concourt à l'éradication des disparités handicapantes, sources de conflits. Cette forme de justice est l'expression même du respect d'autrui, quelle que soit sa puissance, sa place et sa capacité à réagir. C'est une auto-discipline que toutes les entités se devraient d'observer scrupuleusement afin d'éviter d'empiéter dangereusement et douloureusement dans les domaines de définition des autres. Ainsi, on pourrait parvenir à tempérer les ardeurs des uns et à donner confiance aux autres : si la menace pèse sur les premiers du fait de la puissance des seconds, il n'y a aucune possibilité de tranquillité et de paix, et, en conséquence, d'ordre à l'échelle planétaire. La justice dont il s'agit est cette prise en considération de la coexistence entre les entités et donc des lieux communs qui les unissent. À la limite, elle est une conditionnalité qui précède la justice au sens propre. Plus précisément, la justice au figuré, considérée par rapport à celle des tribunaux, est ce que la moralité est à la morale sociale. Étant donné que le bon sens est la chose la mieux partagée, suivant le précepte cartésien, il y a espoir qu'à la lumière de l'expérience acquise, le monde puisse se retrouver sur le meilleur chemin qui soit, du fait de ses différentes composantes, avant tout mécanisme régulateur extérieur ou supérieur.

\section{La place et le rôle prioritaires de l'ONU}

De toute évidence, l'ONU reste l'instrument qui a marqué le siècle dernier, du fait de son rôle depuis la fin de la Deuxième Guerre mondiale. De surcroît, cette organisation, qui regroupe aujourd'hui 192 pays, a consacré son énergie à la résolution de presque tous les conflits depuis sa création, et poursuit son action malgré les difficultés rencontrées. C'est la raison fondamentale qui explique qu'elle a encore une place et un rôle dans le contexte d'un monde en proie au désordre. Aussi, se doit-elle de s'y adapter.

Sans conteste, l'ONU est encore un instrument de relations internationales crédible. C'est le plus grand forum des nations. Elle se saisit des problèmes les plus brûlants qui désorganisent le monde : les tensions, les crises et les conflits. Au-delà de cette saisine, elle intervient sur les théâtres pour le maintien, le rétablissement ou l'imposition de la paix. À ce titre, l'ONU a une place et un rôle prépondérants qu'il faut préserver et améliorer dans le sens de l'efficacité. Mieux, malgré les quasi-revers enregistrés (intervention désastreuse en Somalie, génocide et massacres de masse au Rwanda et en Bosnie-Herzégovine), elle a connu des succès, même limités, ailleurs. Le cas 
le plus édifiant est celui de la Sierra Leone. Dans ce pays d'Afrique de l'Ouest, l'organisation internationale a déployé sa plus grande force de ces dernières années. Elle a accompagné ce pays déchiré dans la voie du rétablissement d'institutions étatiques, y compris l'institution d'un tribunal pour juger les crimes de guerre. Aujourd'hui, l'ONU est présente au Kosovo, en République démocratique du Congo et à Haïti. Cela traduit son utilité. Enfin, ses organismes spécialisés s'investissent dans leur domaine de compétence, à travers le monde, là où le besoin se fait le plus sentir, à la hauteur des moyens disponibles.

Toutefois, l'ONU reste marquée du sceau des grandes puissances entre les mains desquelles sa politique ressemble, à bien des égards, «à une peau de léopard ». Par conséquent, l'organisation devrait s'adapter à l'évolution du monde et se départir des critères qui ont présidé à sa naissance, au rang desquels il y a au moins la grande question du Conseil de sécurité et de sa bureaucratie. À elle seule, cette problématique est un programme. Ainsi, les membres permanents du Conseil de sécurité disposent de prérogatives supérieures, comme le droit de veto. Mais, au moment de la signature de la Charte en 1945, l'idée qui sous-tendait ce droit était le consensus entre les puissances. Or, de plus en plus, le veto est un instrument d'opposition et d'expression des réticences particulières, au nom des intérêts nationaux. Il y a donc la nécessité de réformer en profondeur le Conseil de sécurité et surtout d'élargir la représentativité, en considération de l'évolution du monde, à défaut de donner une force supplémentaire à l'Assemblée générale. En s'attaquant à sa bureaucratie légendaire, l'organisation pourrait améliorer ses prestations et ses résultats. Les types nouveaux de conflits obligent à réadapter la posture. En se créant des liaisons courtes ou des boucles rapides de décision, elle éviterait le fiasco ou les lenteurs dans l'exécution de ses attributions. Le rapport Brahimi ${ }^{1}$ avait déjà donné l'élan qui se concrétise de plus en plus dans le quotidien de l'ONU.

\section{Conclusion}

L'état du monde n'est certainement pas des plus reluisants. La situation est caractérisée par des événements graves qui plongent les citoyens dans l'émoi à travers les dérives, les modes d'action concurrents inadaptés et l'exclusion. De même, la gestion du monde est à l'évidence très perfectible, car elle est chaotique du fait de l'unilatéralisme, des modèles imposés et rejetés ainsi que de l'hypertrophie des intérêts nationaux. En revanche, la résorption de cet état des lieux passe obligatoirement par une thérapie urgente et nécessaire qui tourne autour de la prise en compte des spécificités, d'une justice et d'un rôle central de l'ONU. 
En vertu du principe d'espérance et sur la base des capacités de réaction de l'humanité, l'espoir est permis... car mieux vaut tard que jamais.

\section{Note}

1. Document A/55/305-S/2000/809 du 21 août 2000 .

\section{Références}

Druon, M., 1988, «La Francophonie, ce nouveau nom de l'espérance », in Éthiopiques, $\mathrm{n}^{\circ}$ 50-51, Nouvelle série, Vol. 5, $\mathrm{n}^{\circ}$ 3-4.

Heidegger, M., 1962, Chemins qui ne mènent nulle part, Classique de la philosophie, Editeur NRF, Wolfang Brokmeier.

Huntington, S. P., 1997, Le Choc des civilisations, Odile Jacob, Paris.

Nietzsche, F., 1886, Par-delà bien et mal, Paris: Hachette.

Teixeira, P., 2003, « Le Conseil de sécurité à l'aube du XXIe siècle : quelle volonté et quelle capacité a-t-il de maintenir la paix et la sécurité internationales ?, in Guide du maintien de la paix, sous la direction de Jocelyn Coulon, Outremont, Athéna/CEPES. 